Pak. J. Agri. Sci., Vol. 55(3), 557-566; 2019

ISSN (Print) 0552-9034, ISSN (Online) 2076-0906

DOI: $10.21162 / P A K J A S / 19.7552$

http://www.pakjas.com.pk

\title{
IMPROVING THE PRODUCTIVITY AND SUGAR RECOVERY OF CANE BY POTASH NUTRITION UNDER DIFFERENT PLANTING METHODS
}

\author{
Muhammad Waqas Aslam Cheema ${ }^{1, *}$, Riaz Ahmad ${ }^{1}$, Abdul Khaliq ${ }^{1}$ and Rashid Ahmad ${ }^{1}$ \\ Department of Agronomy, University of Agriculture-38040, Faisalabad, Pakistan \\ "Corresponding author's e-mail: waqasaslamcheema2006@gmail.com
}

\begin{abstract}
Potassium-nutrition is a subject of great consideration, as research has revealed its importance in increasing sugar recovery in sugarcane. While it cannot achieve its genetic expression of yield without proper planting technology. Therefore, the present study was conducted to optimize the planting method and $\mathrm{K}$ level to improve the yield and sugar recovery of sugarcane at University of Agriculture Faisalabad in 2014-2016. Treatments comprised of four planting methods viz. i) $90 \mathrm{~cm}$ spaced pits with a diameter of $90 \mathrm{~cm}$, ii) $90 \mathrm{~cm}$ spaced pits with a diameter of $90 \mathrm{~cm}$ in diagonal fashion, iii) $90 \mathrm{~cm}$ spaced double row strips and iv) $120 \mathrm{~cm}$ spaced trench planting in combination with four $\mathrm{K}$ nutrition levels i.e., 0, 100, 200 and $300 \mathrm{~kg} / \mathrm{ha} \mathrm{K}_{2} \mathrm{O}$. Results revealed that $\mathrm{K}$ application improved the growth, cane yield and sugar recovery irrespective of planting method. However, maximum number of tillers were recorded in sugarcane sown in diagonal pit planting at $90 \mathrm{~cm}$ with $100 \mathrm{~kg} / \mathrm{ha} \mathrm{K}_{2} \mathrm{O}$. Likewise, Leaf area index and net assimilation rate were substantially improved with $\mathrm{K}$ application in all planting methods. More cane weight (15\%) was obtained at sugarcane sown in $120 \mathrm{~cm}$ spaced trenches with $200 \mathrm{~kg} / \mathrm{ha} \mathrm{K}_{2} \mathrm{O}$ during both the years. Stripped cane yield was maximum in diagonal pit planting at $90 \mathrm{~cm}$ with $100 \mathrm{~kg} / \mathrm{ha} \mathrm{K}_{2} \mathrm{O}(113.7 \mathrm{t} / \mathrm{ha})$ during the plant crop year and diagonal pit plantation $+200 \mathrm{~kg} / \mathrm{ha} \mathrm{K}_{2} \mathrm{O}(98.22 \mathrm{t} / \mathrm{ha}$ ) during the ratoon crop year. Likewise, maximum potassium use efficiency (KUE) i.e. 99.5 and $88.2 \mathrm{~kg} / \mathrm{kg}$ were recorded in planting of sugarcane in diagonal pits at $90 \mathrm{~cm}$ with $100 \mathrm{~kg} / \mathrm{ha}$ $\mathrm{K}_{2} \mathrm{O}$ during plant crop year and $120 \mathrm{~cm}$ trenches $+100 \mathrm{~kg} / \mathrm{ha} \mathrm{K}_{2} \mathrm{O}$ during the ratoon crop year, respectively. Sugar recovery was also enhanced by all the $\mathrm{K}$ nutrition levels over control. The cultivation of sugarcane in $90 \mathrm{~cm}$ spaced pits with the supplementation of potash at $100 \mathrm{~kg} / \mathrm{ha}$ gave the maximum sugar yield of $15.8 \mathrm{t} / \mathrm{ha}$ in plant crop year and $13.2 \mathrm{t} / \mathrm{ha}$ in the ratoon crop year. The combined economic analysis over two years (plant + ratoon year) revealed that sugarcane planting was more beneficial at $120 \mathrm{~cm}$ spaced trenches, with $100 \mathrm{~kg}$ potash/ha $(3678 \$)$; which was followed by $90 \mathrm{~cm}$ diagonal pit plantation $+100 \mathrm{~kg} / \mathrm{ha} \mathrm{K} \mathrm{K}_{2} \mathrm{O}$ which gave the combined benefits of 3611 . Sugar cane may be planted in $90 \mathrm{~cm}$ diagonal pits and $120 \mathrm{~cm}$ spaced trenches with $100 \mathrm{~kg} / \mathrm{ha}$ potash to improve the cane yield and sugar recovery.
\end{abstract}

Keywords: Sugarcane, planting methods, potash nutrition, sugar recovery

\section{INTRODUCTION}

Sugarcane (Saccharum officinarum L.) is major cash crop of Pakistan, as it provides raw materials to sugar and allied industries, while it contributes about $3.2 \%$ in value addition product to country's GDP. In Pakistan, sugarcane is being cultivated on more than 1.2 million hectares with average yield of about $60 \mathrm{t} / \mathrm{ha}$, far less than potential yield of $120 \mathrm{t} / \mathrm{ha}$ (Govt. of Pakistan, 2016). This yield gap is due to many factors like unapproved varieties, improper planting methods, imbalance fertilization, sugarcane pest especially borers, diseases like red rot, drought and lodging issues (Abbas et al., 2016) but among these, major two factors are the imbalance fertilization especially use of potassium (K) fertilizer and improper planting methods (Ehsanullah et al., 2011; Ghaffar et al., 2012; Ali et al., 2018).

Sugarcane is a highly exhaustive crop and it mines large amounts of nitrogen, phosphorus and potassium nutrients from the soil for its growth. It has been found that sugarcane extracts about $205 \mathrm{~kg} \mathrm{~N}, 55 \mathrm{~kg} \mathrm{P}_{2} \mathrm{O}_{5}, 275 \mathrm{~kg} \mathrm{~K} 2 \mathrm{O}, 30 \mathrm{~kg} \mathrm{~S}$,
$3.5 \mathrm{~kg} \mathrm{Fe}, 1.2 \mathrm{~kg} \mathrm{Mn}, 0.6 \mathrm{~kg} \mathrm{Zn}$ and $0.2 \mathrm{~kg} \mathrm{Cu}$ from the soil for a cane yield of $100 \mathrm{t} / \mathrm{ha}$ (Singh, 2007). Higher extraction of $\mathrm{K}$ by sugarcane is related to its synthesis and translocation of sucrose from leaves to the storage tissues in stalks (El-Tilib et al., 2004), helps in phloem loading and unloading (Shukla et al., 2009) and augments the activity of $\mathrm{NO}_{3}{ }^{-1}$ which increases the nitrogen uptake (Chohan et al., 2013). Potassium is not only essential for sucrose activity but it also promotes resistance to pests and disease in sugarcane (Krauss, 2001). Hunsigi (2001) observed that $\mathrm{K}$ application helps sugarcane crop to reach maturity. Thus, the addition of adequate potash nutrition is necessary.

The second major issue in cane production is inappropriate geometrical arrangement or planting patterns. Farmers have been using flat sowing method of sugarcane since decades. Although this method of sugarcane plantation is easy to practice but it leads to poor sprouting (30-35\%) of sugarcane setts due to unavailability of adequate moisture from of the soil (Singh, 2002). Moreover, vegetative growth is less in closed spacing (Smit and Singels, 2006). Hence, there was a 
dire need for new and promising planting patterns in sugarcane to attain better yield.

New techniques of sugarcane plantation are getting popular since last decade these include pit plantation of sugarcane, trench plantation, double strip sowing of sugarcane (Chattha et al., 2007), ring pit method and chip bud technology (Jain et al., 2006). All these planting methods are aimed to improve the growth and biomass production (Bashir and Saeed, 2000) with higher sugar recovery (Singh et al., 2009) and have the advantage of being mechanically operated which reduces the labor cost, increase precision and ultimately increasing the stripped cane yield and sugar recovery with greater net field benefits (Nalewade et al., 2018). There are many studies available regarding the effect of planting method and $\mathrm{K}$ application on growth, yield and sugar recovery of sugarcane. However, the interaction of different planting methods with $\mathrm{K}$ nutrition on sugar recovery and KUE has rarely been studied. Therefore, the present study was conducted to optimize the $\mathrm{K}$ application in different planting methods to improve the growth, cane yield, sugar recovery and KUE of sugarcane. The specific objective of this study was to evaluate the economics of $\mathrm{K}$ fertilization in combination with different planting methods in sugarcane.

\section{MATERIALS AND METHODS}

Experimental site and treatments: A field experiment was conducted for two consecutive years during 2013-14 and 2015-16 at Agronomic Research Area, Department of
Agronomy, University of Agriculture, Faisalabad $\left(1.25^{\circ} \mathrm{N}\right.$ latitude, $73.09^{\circ} \mathrm{E}$ longitude, altitude $\left.184 \mathrm{~m}\right)$. After the first harvest (2013-14), the second crop (2015-16) was kept as ratoon. The experimental soil was sandy loam with EC, 0.34 , pH 8.20 , organic matter $0.72 \%$ and nitrogen $0.03 \%$ while extractable phosphorus (5.8 ppm), potassium (140 ppm) and DTPA extractable Zn (0.89).

Experimental treatments were executed in randomized complete block design (RCBD) in split plot arrangements. The planting patterns, i.e. $90 \mathrm{~cm}$ spaced pits with diameter 90 $\mathrm{cm}$ (90 by 90 pits), $90 \mathrm{~cm}$ spaced pits with diameter $90 \mathrm{~cm}$ in diagonal fashion (90 by $90 \mathrm{~d}$-pits), $90 \mathrm{~cm}$ spaced double row strips (90 SDRS) and $120 \mathrm{~cm}$ spaced trenches (120 ST) were kept in main plots whilst potassium levels i.e., 0, 100, 200 and $300 \mathrm{~kg} / \mathrm{ha} \mathrm{K}_{2} \mathrm{O}$ were kept in subplots. The plot size for $90 \mathrm{~cm}$ spaced pits, Diagonal pit plantation and $120 \mathrm{~cm}$ spaced pits was $5.4 \mathrm{~m} \times 8 \mathrm{~m}$ whereas plot size for $90 \mathrm{~cm}$ spaced double row strips was $3.6 \mathrm{~m} \times 8 \mathrm{~m}$.

Field preparation: During 2013-14, for plant crop, the land was leveled by laser land leveler. Disk plough and disk harrow were used for pulverizing the soil followed by planking. Pits $(90 \mathrm{~cm}$ in depth and $90 \mathrm{~cm}$ apart in distance from either of four sides) were made mechanically by pit hole digger operated by $75 \mathrm{HP}$ tractor. Pits were then irrigated with $100 \mathrm{~mm}$ water to settle down the loose soil. Similar procedures were adopted while digging $90 \mathrm{~cm}$ spaced pits of the diameter of $90 \mathrm{~cm}$ in a diagonal arrangement in this system except a fifth pit was also dug in between these four

(b)

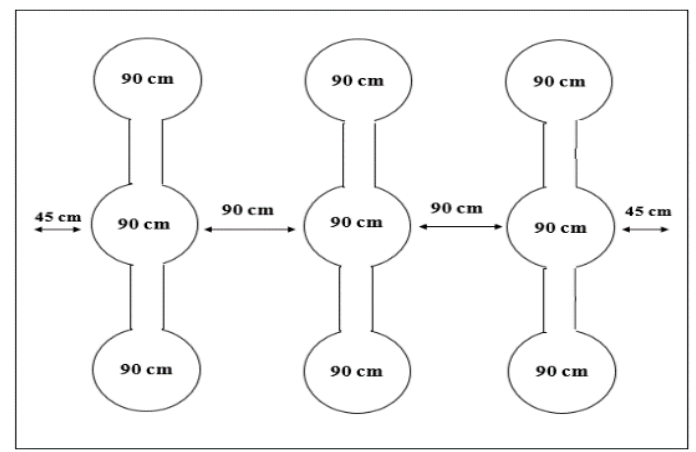

(d)

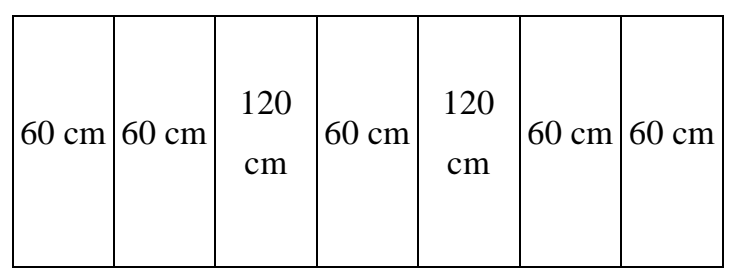

Figure 1.Schematic diagram of different planting patterns: (a) Diagonal pit plantation of sugarcane in $90 \mathrm{~cm}(\mathrm{~b}) 90$ cm spaced pit plantation (c) $90 \mathrm{~cm}$ spaced double row strips (d) $120 \mathrm{~cm}$ spaced trenches. 
pits. Trenches at the uniform spacing of $120 \mathrm{~cm}$ were made using tractor mounted ridger.

Crop husbandry: The sugarcane variety 2003-USS-127 was planted on $7^{\text {th }}$ October 2013. This variety is originated from canal point Florida, USA which is recommended for lodging resistant because of greater fiber contents and also has adequate sugar recovery level. Fertilizers were applied at the rate of $175 \mathrm{~kg} \mathrm{~N}, 112 \mathrm{~kg} \mathrm{P}_{2} \mathrm{O}_{5}$, while $\mathrm{K}$ was applied according to the experimental treatments, however, recommended dose of potassium for sugarcane is $112 \mathrm{~kg} / \mathrm{ha}$. All of phosphorous, potash and half of the nitrogen were applied at the time of sowing in the form of DAP (diammonium phosphate), SOP (sulphate of potash) and urea, respectively. Remaining nitrogen was equally applied in splits at tillering and grand growth stages. For the ratoon crop, about 30\% more phosphorus and nitrogen fertilizer were applied. Potash nutrition treatments were applied according to the treatment plan. In total 64-acre-inches irrigation water was applied from sowing to harvesting of the crop during both years in addition to rainfall. Chlorpyriphos at $2.5 \mathrm{~L} / \mathrm{ha}$ solution for the prevention of red rot and termite attack was applied at sowing time. Carbofuran (3\% G) granules at $35 \mathrm{~kg} / \mathrm{ha}$ were applied for the purpose of controlling borer attack in sugarcane crop. The weeds were controlled manually.

Crop harvest: The plant crop was harvested when it reached physiological maturity on $10^{\text {th }}$ of March 2015, after it was kept as ratoon crop for the second-year trial. The ratoon crop ( $2^{\text {nd }}-$ year trial) was harvested on $21^{\text {st }}$ February 2016. The total duration of Planted crop was 14 months (60 weeks), however, the time for the ratoon crop was 11 months (50 weeks).

\section{Observations}

Morphological and yield traits: Germination or sprouting percentage was calculated according to following formula.

$$
\text { Germination percentage }=\frac{\text { No. of buds germinated }}{\text { Total no. of buds }} \times 100
$$

Number of sprouts or tillers $/ \mathrm{m}^{2}$ was calculated at 90 days after sowing. The internodal length was measured by selecting random 10 stripped canes from each plot and lengths were measured in $\mathrm{cm}$ and then averaged. Leaf area index was calculated by following formula.

Leaf area index $(L A I)=$ Leaf area/land area .

Leaf area duration was calculated by the formula given by Hunt (1978).

$$
\text { Leaf area duration }(\mathrm{LAD})=\frac{\mathrm{LAI}_{1}+\mathrm{LAI}_{2}}{2} \times\left(\mathrm{t}_{2}-\mathrm{t}_{1}\right)
$$

$\mathrm{LAI}_{1}$ describes the leaf area index of first 30 days. $\mathrm{LAI}_{2}$ describes the leaf area index taken after 60 days at $2^{\text {nd }}$ time. While net assimilation was measured by using the following formula by Hunt (1978).

$$
\text { Net Assimilation Rate }(\mathrm{NAR})=\frac{\mathrm{TDM}}{\mathrm{LAD}}
$$

Cane weight was calculated by weighing 20 single canes from each plot and then averaged. Number of millable canes per unit area was calculated by counting the total number of canes from each treatment and then presented on $\mathrm{m}^{2}$ basis. Stripped cane yield was obtained by weighing all stripped canes. Agronomic potassium use efficiency was calculated by following formula (SSSP, 1994).

Agronomic $\mathrm{K}^{+}$use efficency

$$
=\frac{\text { Stripped cane yield }(\mathrm{F})-\text { Stripped cane yield }(\mathrm{C})}{\text { Fertilizer Nutrient applied }(\mathrm{K})}
$$

Stripped cane yield $(\mathrm{F})=$ stripped cane yield when crop was fertilized, Stripped cane yield $(\mathrm{C})=$ stripped cane yield where no fertilizer was applied (control)

Sugar quality traits: Commercial cane sugar was calculated by selecting 10 canes from each plot and finding out the value of Brix \% by hydrometer. Polarity \% was measured by polarity meter of cane juice extracted from those 10 canes and then putting these values in formula given by (Spenser and meads, 1963).

Commercial Cane Sugar $(\mathrm{CCS} \%)=\frac{3}{2} \mathrm{P}\left[1-\mathrm{F}+\frac{5}{100}\right]-$
\[ \frac{1}{2} \mathrm{~B}\left[1-\mathrm{F}+\frac{3}{10}\right] \]

$$
\frac{1}{2} \mathrm{~B}\left[1-\mathrm{F}+\frac{3}{100}\right]
$$

Total sugar recovery was calculated by multiplying commercial cane sugar with 0.94 (correction factor). Total sugar yield was determined by following formula.

Sugar yield (t/ha)

$=\frac{\text { Stripped cane yield }(\mathrm{t} / \mathrm{ha}) \times \text { Commercial Cane sugar }(\mathrm{CCS})}{100}$

Statistical and economic analysis: Data collected from this experiment were statistically analyzed by computer package STATISTIX 8.2 (analytical software, Tallahassee, FL, USA) and differences among the treatment means were compared by using least significant difference (LSD) test at 5\% probability (Steel et al., 1997) Economic and marginal analyses were carried out according to the principles laid out by Byrelee (CIMMYT, 1988).

\section{RESULTS}

Results revealed that planting methods did not significantly affect the tillers during first crop year while results were significant during the second year of study. Moreover, K application significantly affected the number of tillers during both the years. Interaction of planting method and $\mathrm{K}$ application was also significant for number of tillers during both study years. Maximum number of tillers $/ \mathrm{m}^{2}$ were recorded for $120 \mathrm{ST}$ and trench planting at $100 \mathrm{~kg} \mathrm{~K}_{2} \mathrm{O}$ and $90 \mathrm{SDRS}$ at $300 \mathrm{~kg} \mathrm{~K} \mathrm{~K}_{2} \mathrm{O}$ during plant crop year, whereas during ratoon crop year it was maximum in 90 by 90 pits with $100 \mathrm{~kg} \mathrm{~K}_{2} \mathrm{O}$ application. Potassium application has a positive effect on the internodal distance of sugarcane during both plant crop and ratoon crop year. However, with the increase of $\mathrm{K}_{2} \mathrm{O}$ application beyond $100 \mathrm{~kg} \mathrm{ha}^{-1}$, no significant effect was recorded. In ratoon crop year a linear increase in internodal length was recorded up to $200 \mathrm{~kg} / \mathrm{ha} \mathrm{K}_{2} \mathrm{O}$ (Table 1). The internodal length was significantly affected by planting patterns as maximum length during plant crop was recorded in 90 by $90 \mathrm{~d}$-pits $(13.99 \mathrm{~cm})$ while maximum 
Cheema, Ahmad, Khaliq \& Ahmad

Table 1. Number of tillers and internodal length as affected by various planting methods and $K$ application rates.

\begin{tabular}{|c|c|c|c|c|c|c|c|c|c|c|}
\hline \multirow[t]{4}{*}{ Planting methods } & \multicolumn{6}{|c|}{ 2014-15 (Planted crop) } & \multicolumn{3}{|c|}{ 2015-16 (Ratoon crop) } & \multirow[b]{3}{*}{ Mean (P) } \\
\hline & \multicolumn{9}{|c|}{ Potash application } & \\
\hline & $\mathbf{0}$ & $100 \mathrm{~kg} \mathrm{ha}^{-1}$ & $200 \mathrm{~kg} \mathrm{ha}^{-1}$ & $300 \mathrm{~kg} \mathrm{ha}^{-1}$ & Mean (P) & 0 & $100 \mathrm{~kg} \mathrm{ha}^{-1}$ & $200 \mathrm{~kg} / \mathrm{ha}^{-1}$ & $300 \mathrm{~kg} \mathrm{ha}^{-1}$ & \\
\hline & \multicolumn{10}{|c|}{ Number of tillers /sprouts $\mathbf{m}^{-2}$} \\
\hline Pit planting@90 & $12.51 \mathrm{~d}$ & $14.28 \mathrm{ab}$ & $13.68 \mathrm{a}-\mathrm{d}$ & $14.91 \mathrm{a}$ & 13.85 & $12.80 \mathrm{~d}$ & $13.43 \mathrm{a}-\mathrm{d}$ & $13.17 \mathrm{~cd}$ & $13.17 \mathrm{~cd}$ & $13.14 \mathrm{~B}$ \\
\hline Diagonal pits@90 & $12.74 \mathrm{~cd}$ & $14.69 \mathrm{a}$ & $14.29 \mathrm{ab}$ & $14.06 \mathrm{abc}$ & 13.94 & $13.20 \mathrm{bcd}$ & $14.16 \mathrm{a}$ & $13.56 a-d$ & $13.67 \mathrm{abc}$ & $13.64 \mathrm{~A}$ \\
\hline Double row strips & $12.86 \mathrm{~cd}$ & $14.35 \mathrm{ab}$ & $13.77 \mathrm{a}-\mathrm{d}$ & $14.77 \mathrm{a}$ & 13.94 & $13.57 \mathrm{a}-\mathrm{d}$ & $13.97 \mathrm{ab}$ & $13.93 \mathrm{abc}$ & $13.70 \mathrm{abc}$ & $13.79 \mathrm{~A}$ \\
\hline Trenches@120cm & $13.29 \mathrm{bcd}$ & $14.79 \mathrm{a}$ & $13.94 \mathrm{abc}$ & $13.68 \mathrm{a}-\mathrm{d}$ & 13.93 & $12.80 \mathrm{~d}$ & $13.93 \mathrm{abc}$ & $13.70 \mathrm{abc}$ & $13.23 \mathrm{bcd}$ & $13.42 \mathrm{AB}$ \\
\hline Mean $(\mathrm{K})$ & $12.85 \mathrm{~B}$ & $14.53 \mathrm{~A}$ & $13.92 \mathrm{~A}$ & $14.36 \mathrm{~A}$ & & $13.09 \mathrm{C}$ & $13.87 \mathrm{~A}$ & $13.59 \mathrm{AB}$ & 13.44BC & \\
\hline $\mathrm{LSD} \leq 0.05$ & \multicolumn{5}{|c|}{$\mathrm{K}=0.68 ; \mathrm{P}=0.58 ; \mathrm{P} . \mathrm{A} \times \mathrm{P} . \mathrm{M}=1.30$} & \multicolumn{5}{|c|}{$\mathrm{K}=0.38 ; \mathrm{P}=0.39 ; \mathrm{K} \times \mathrm{P}=0.82$} \\
\hline \multicolumn{11}{|c|}{ Internodal length $(\mathrm{cm})$} \\
\hline Pit planting@90 & $11.61 \mathrm{e}$ & $13.49 \mathrm{bcd}$ & $13.33 \mathrm{bcd}$ & $13.33 \mathrm{bcd}$ & $12.94 \mathrm{~B}$ & $13.23 \mathrm{fg}$ & $13.75 \mathrm{a}$ & $13.77 \mathrm{a}$ & $13.62 \mathrm{a}-\mathrm{d}$ & $13.59 \mathrm{~A}$ \\
\hline Diagonal pits@90 & 11.98de & $16.38 \mathrm{a}$ & $14.34 \mathrm{~b}$ & $13.26 \mathrm{bcd}$ & $13.99 \mathrm{~A}$ & $13.35 \mathrm{efg}$ & $13.40 \mathrm{~d}-\mathrm{g}$ & $13.64 \mathrm{abc}$ & $13.35 \mathrm{efg}$ & $13.43 \mathrm{~B}$ \\
\hline Double row strips & $12.17 \mathrm{de}$ & $12.83 b-e$ & $12.54 \mathrm{cde}$ & $12.97 \mathrm{~b}-\mathrm{e}$ & $12.63 \mathrm{~B}$ & $13.50 \mathrm{~b}-\mathrm{e}$ & $13.69 \mathrm{ab}$ & $13.74 \mathrm{a}$ & $13.58 \mathrm{a}-\mathrm{d}$ & $13.63 \mathrm{~A}$ \\
\hline Trenches@120cm & $12.89 \mathrm{~b}-\mathrm{e}$ & $13.58 \mathrm{bcd}$ & $13.81 \mathrm{bc}$ & $13.36 \mathrm{bcd}$ & $13.41 \mathrm{AB}$ & $13.18 \mathrm{~g}$ & $13.48 \mathrm{~b}-\mathrm{e}$ & $13.55 \mathrm{a}-\mathrm{e}$ & $13.44 \mathrm{c}-\mathrm{f}$ & $13.41 \mathrm{~B}$ \\
\hline Mean $(\mathrm{K})$ & $12.16 \mathrm{C}$ & $14.07 \mathrm{~A}$ & $13.50 \mathrm{AB}$ & $13.23 \mathrm{~B}$ & & $13.32 \mathrm{C}$ & $13.58 \mathrm{AB}$ & $13.6 \mathrm{~A}$ & $13.50 \mathrm{~B}$ & \\
\hline $\mathrm{LSD} \leq 0.05$ & \multicolumn{5}{|c|}{$\mathrm{K}=0.72 ; \mathrm{P}=0.99 ; \mathrm{P} . \mathrm{A} \times \mathrm{P} . \mathrm{M}=1.59$} & \multicolumn{5}{|c|}{$\mathrm{K}=0.10 ; \mathrm{P}=0.14 ; \mathrm{K} \times \mathrm{P}=0.23$} \\
\hline
\end{tabular}

\begin{tabular}{lcccc}
\hline Trend analysis for potash nutrition treatments & Number of sprouts (tillers) & \multicolumn{2}{c}{ Internodal length (cm) } \\
\cline { 2 - 5 } & $\mathbf{2 0 1 4}$ & $\mathbf{2 0 1 5}$ & $\mathbf{2 0 1 4}$ & $\mathbf{2 0 1 5}$ \\
\hline Linear & $*$ & $\mathrm{Ns}$ & $*$ & $*$ \\
Quadratic & $*$ & $*$ & $*$ & $*$ \\
Cubic & $*$ & $\mathrm{Ns}$ & $\mathrm{ns}$ \\
\hline
\end{tabular}

$\mathrm{K}=$ Potash application; $\mathrm{P}=$ Planting methods; *=Significant $(\mathrm{P}<0.05)$; ns =Non-significant; Capital alphabetical letters (A, B, C, D) with mean values represent the significant differences for main effects, while small alphabetical letters $(a, b, c .$.$) represent the interaction$ between planting methods and potash nutrition.

internodal length was attained in 90 SDRS $(13.63 \mathrm{~cm})$ during the ratoon crop year (Table 1).

Leaf area index showed a linear increase in response to potash application up to the levels of $200 \mathrm{~kg} \mathrm{ha}^{-1}$ (6.91) during both the years, however, the addition of more potash $(300 \mathrm{~kg} / \mathrm{ha})$ did not significantly increase the leaf area index. Similar trends were observed by cumulative leaf area duration and net assimilation rate in response to $\mathrm{K}_{2} \mathrm{O}$ application (Table 2). Planting methods had no significant effect on leaf area index during the plant crop year but during the ratoon crop year, 90 by 90 pits (6.53) were best among all other planting methods in increasing leaf area index. Interaction of 90 by 90 pits and $200 \mathrm{~kg} / \mathrm{ha}$ (7.02 during the plant crop and 6.70 during the ratoon crop year) has increased the leaf area index to a maximum extent during both years of study. Planting methods also significantly affected leaf area duration and net assimilation rate. It was found that 90 by 90 pits produced the maximum leaf area duration during the plant crop year (1002 days) and ratoon crop year (965 days), whereas 90 by $90 \mathrm{~d}$ pits provided the highest value for the net assimilation rate during plant crop year $\left(3.46 \mathrm{~kg} \mathrm{~m}^{-2}\right.$ day $\left.^{-1}\right)$ and ratoon crop year (3.16 kg $/ \mathrm{m}^{2} /$ day) (Table 2 ).

A significant increase in cane weight was recorded by the application of $100 \mathrm{~kg} \mathrm{~K}_{2} \mathrm{O} \mathrm{ha}^{-1}(0.92 \mathrm{~kg}$ ) which is statistically at par with the application of $200 \mathrm{~kg} / \mathrm{ha} \mathrm{K}_{2} \mathrm{O}(0.9 \mathrm{~kg})$ and 300 $\mathrm{kg} / \mathrm{ha} \mathrm{K}_{2} \mathrm{O}(0.90 \mathrm{~kg})$ during the plant crop year whereas a linear increase was observed in cane weight up to $200 \mathrm{~kg} / \mathrm{ha}$
$\mathrm{K}_{2} \mathrm{O}(0.89 \mathrm{~kg})$ (Table 3). Increase in application beyond 200 $\mathrm{kg} / \mathrm{ha}$ had a substantial inverse effect on cane weight during the ratoon crop year. Amongst the planting methods, 120 ST produced healthier canes with $11-16 \%$ and $2-6 \%$ more cane weight than that of others during plant crop and ratoon crop year. The sugarcane plantation at $120 \mathrm{~cm}$ trenches with 200 $\mathrm{kg} / \mathrm{ha}$ potash nutrition improved cane weight during both years $(1.03 \mathrm{~kg}$ during plant crop year and $0.93 \mathrm{~kg}$ during the ratoon crop year). Number of millable canes was significantly influenced by the application of potash nutrition during both plant crop and ratoon crop year. The increase of about 9-10\% in plant population in comparison with control was observed in the plant crop year. Overall millable canes were 6-8\% less in ratoon crop year than that of plant crop year. In the planting patterns, 90 by $90 \mathrm{~d}$-pits $\left(12.57\right.$ canes $/ \mathrm{m}^{2}$ during plant crop year and 11.20 canes $/ \mathrm{m}^{2}$ during the ratoon crop year) resulted in more production of millable canes than all other planting patterns (Table 3 ). The best combination was observed with 90 by $90 \mathrm{~d}$-pits at $200 \mathrm{~kg} \mathrm{ha}^{-1}$ potassium application during plant crop year $(12.83$ canes/ $\mathrm{m})$ and 90 by $90 \mathrm{~d}$-pits at $100 \mathrm{~kg}$ $\mathrm{K}_{2} \mathrm{O} \mathrm{ha}^{-1}$ during the ratoon crop year $\left(11.55\right.$ canes $\left./ \mathrm{m}^{2}\right)$.

Stripped cane yield was increased during plant crop and ratoon crop year by the application of potash nutrition (Table 4). However, 90 by $90 \mathrm{~d}$-pits proved to be the best planting method amongst all other in producing the striped cane yield during both the years with 106.95 and $96.42 \mathrm{t} / \mathrm{ha}$ yield, respectively. 
Table 2. Leaf area index, leaf area duration and Net assimilation rate as affected by various planting methods and $\mathrm{K}$ application rates.

\begin{tabular}{|c|c|c|c|c|c|c|c|c|c|c|}
\hline \multirow[t]{3}{*}{ planting methods } & \multicolumn{5}{|c|}{ 2014-15 (Planted crop) } & \multicolumn{5}{|c|}{ 2015-16 (Ratoon crop) } \\
\hline & \multicolumn{10}{|c|}{ Potash application } \\
\hline & $\mathbf{0}$ & $100 \mathrm{~kg} \mathrm{ha}^{-1}$ & $200 \mathrm{~kg} \mathrm{ha}^{-1}$ & $300 \mathrm{kgha}^{-1}$ & Mean (P) & 0 & 100 kgha $^{-1}$ & $200 \mathrm{~kg} / \mathrm{ha}^{-1}$ & 300 kgha $^{-1}$ & Mean (P) \\
\hline & \multicolumn{10}{|c|}{ Maximum leaf area index } \\
\hline Pit planting at 90 & $6.35 f$ & $6.76 a-e$ & $7.02 \mathrm{a}$ & $6.49 \mathrm{def}$ & 6.66 & $6.15 \mathrm{~cd}$ & $6.55 \mathrm{ab}$ & $6.70 \mathrm{a}$ & $6.73 a$ & $6.53 \mathrm{~A}$ \\
\hline Diagonal pits@90 & $6.79 a-e$ & $6.77 \mathrm{a}-\mathrm{e}$ & $6.89 \mathrm{abc}$ & $6.73 a-f$ & 6.80 & $5.89 \mathrm{~d}$ & $6.52 \mathrm{ab}$ & $6.51 \mathrm{ab}$ & $6.39 \mathrm{abc}$ & $6.33 \mathrm{AB}$ \\
\hline Double row strips & $6.44 \mathrm{ef}$ & $6.54 b-f$ & $6.84 a-d$ & $6.79 \mathrm{a}-\mathrm{e}$ & 6.65 & $5.33 \mathrm{e}$ & $6.28 \mathrm{bc}$ & $6.53 \mathrm{ab}$ & $6.35 \mathrm{bc}$ & $6.12 \mathrm{~B}$ \\
\hline Trenches@120cm & $6.50 c-f$ & $6.77 \mathrm{a}-\mathrm{e}$ & $6.90 \mathrm{ab}$ & $6.68 \mathrm{a}-\mathrm{f}$ & 6.71 & $5.53 \mathrm{e}$ & $6.29 b c$ & $6.45 \mathrm{abc}$ & $6.41 \mathrm{abc}$ & $6.17 \mathrm{~B}$ \\
\hline Mean $(\mathrm{K})$ & $6.52 \mathrm{C}$ & $6.71 \mathrm{~B}$ & $6.91 \mathrm{~A}$ & $6.67 \mathrm{BC}$ & & $5.73 \mathrm{C}$ & $6.41 \mathrm{~B}$ & $6.55 \mathrm{~A}$ & $6.47 \mathrm{AB}$ & \\
\hline \multirow[t]{2}{*}{$\mathrm{LSD} \leq 0.05$} & \multicolumn{5}{|c|}{$\mathrm{K}=0.24 ; \mathrm{P}=0.18 ; \mathrm{K} \times \mathrm{P}=0.39$} & \multicolumn{5}{|c|}{$\mathrm{K}=0.14 ; \mathrm{P}=0.25 ; \mathrm{K} \times \mathrm{P}=0.34$} \\
\hline & \multicolumn{10}{|c|}{ Cumulative leaf area duration } \\
\hline Pit planting@90 & $983.91 b c$ & $1001.80 \mathrm{bc}$ & $1027.01 \mathrm{a}$ & 998.24 & $1002.74 \mathrm{~A}$ & $934.02 \mathrm{de}$ & $980.98 \mathrm{ab}$ & $982.99 a$ & $965.34 \mathrm{abc}$ & $965.83 \mathrm{~A}$ \\
\hline Diagonal pits@90 & $925.54 d$ & $986.98 \mathrm{bc}$ & $996.80 \mathrm{bc}$ & $995.66 b c$ & $976.24 \mathrm{~B}$ & $879.14 \mathrm{f}$ & 978.15 & $988.81 \mathrm{a}$ & $973.74 \mathrm{abc}$ & $954.96 \mathrm{~A}$ \\
\hline Double row strips & $942.23 \mathrm{~d}$ & $981.29 \mathrm{c}$ & $993.94 b c$ & $991.69 b c$ & 977.29B & $815.99 \mathrm{~g}$ & 900.06 & $970.73 \mathrm{abc}$ & 928.66de & $903.86 \mathrm{~B}$ \\
\hline Trenches@120cm & $936.99 \mathrm{~d}$ & $1000.21 b c$ & $1006.69 \mathrm{ab}$ & $989.07 b c$ & 983.24B & $894.13 f$ & $943.91 \mathrm{~cd}$ & $987.60 \mathrm{a}$ & $948.40 \mathrm{bcd}$ & $943.51 \mathrm{~A}$ \\
\hline Mean $(\mathrm{K})$ & $947.17 \mathrm{C}$ & $992.57 \mathrm{~B}$ & $1006.11 \mathrm{~A}$ & $993.66 \mathrm{~B}$ & & $880.82 \mathrm{C}$ & $950.78 \mathrm{~B}$ & $982.53 \mathrm{~A}$ & 954.04B & \\
\hline \multirow[t]{2}{*}{$\mathrm{LSD} \leq 0.05$} & \multicolumn{5}{|c|}{$\mathrm{K}=10.27 ; \mathrm{P}=15.27 ; \mathrm{K} \times \mathrm{P}=23.95$} & \multicolumn{5}{|c|}{$\mathrm{K}=14.53 ; \mathrm{P}=23.16 ; \mathrm{K} \times \mathrm{P}=34.25$} \\
\hline & \multicolumn{10}{|c|}{ Net assimilation rate } \\
\hline Pit planting@90 & 2.94 & $3.22 \mathrm{c}$ & $3.23 \mathrm{c}$ & $3.08 \mathrm{~d}$ & $3.12 \mathrm{~B}$ & $2.87 \mathrm{ef}$ & $2.95 \mathrm{de}$ & $2.90 \mathrm{ef}$ & $2.95 \mathrm{de}$ & $2.92 \mathrm{~B}$ \\
\hline Diagonal pits@90 & $3.43 b$ & $3.58 \mathrm{a}$ & $3.45 b$ & $3.38 \mathrm{~b}$ & $3.46 \mathrm{~A}$ & $3.07 \mathrm{bcd}$ & $3.15 b$ & $3.31 \mathrm{a}$ & $3.11 b c$ & $3.16 \mathrm{~A}$ \\
\hline Double row strips & $2.50 \mathrm{f}$ & $2.55 \mathrm{f}$ & $2.52 \mathrm{f}$ & $2.58 \mathrm{f}$ & $2.53 \mathrm{C}$ & $2.47 \mathrm{~h}$ & $2.64 \mathrm{~g}$ & $2.80 \mathrm{f}$ & $2.56 \mathrm{gh}$ & $2.62 \mathrm{C}$ \\
\hline Trenches@120cm & $3.11 \mathrm{~d}$ & $3.17 \mathrm{~cd}$ & $3.18 \mathrm{~cd}$ & $3.12 \mathrm{~cd}$ & $3.15 \mathrm{~B}$ & $2.90 \mathrm{ef}$ & $3.09 \mathrm{bcd}$ & $2.97 \mathrm{cde}$ & $2.99 \mathrm{cde}$ & $2.99 \mathrm{~B}$ \\
\hline Mean $(\mathrm{K})$ & $2.99 \mathrm{C}$ & $3.13 \mathrm{~A}$ & $3.09 \mathrm{AB}$ & 3.04BC & & $2.83 \mathrm{C}$ & $2.96 \mathrm{AB}$ & $3.00 \mathrm{~A}$ & $2.90 \mathrm{AB}$ & \\
\hline $\mathrm{LSD} \leq 0.05$ & \multicolumn{5}{|c|}{$\mathrm{K}=0.05 ; \mathrm{P}=0.06 ; \mathrm{K} \times \mathrm{P}=0.17$} & \multicolumn{5}{|c|}{$\mathrm{K}=0.27 ; \mathrm{P}=0.44 ; \mathrm{K} \times \mathrm{P}=0.67$} \\
\hline
\end{tabular}

Table 3. Cane weight $(\mathrm{kg})$ and number of millable cane $\left(\mathrm{m}^{2}\right)$ as affected by various planting methods and $\mathrm{K}$ application rates.

\begin{tabular}{|c|c|c|c|c|c|c|c|c|c|c|}
\hline \multirow[t]{3}{*}{ planting methods } & \multicolumn{5}{|c|}{ 2014-15 (Plant crop) } & \multicolumn{5}{|c|}{ 2015-16(Ratoon crop) } \\
\hline & \multicolumn{10}{|c|}{ Potash application } \\
\hline & $\mathbf{0}$ & $100 \mathrm{~kg} \mathrm{ha}^{-1}$ & $200 \mathrm{~kg} \mathrm{ha}^{-1}$ & 300 kgha $^{-1}$ & Mean (P) & $\mathbf{0}$ & 100 kgha $^{-1}$ & $200 \mathrm{~kg} / \mathrm{ha}^{-1}$ & 300 kgha $^{-1}$ & Mean (P) \\
\hline & \multicolumn{10}{|c|}{ Cane weight (kg) } \\
\hline Pit planting@90 & 0.87 efg & $0.91 \mathrm{~d}$ & $0.88 \mathrm{ef}$ & $0.91 \mathrm{~d}$ & $0.89 \mathrm{~B}$ & $0.87 \mathrm{bcd}$ & $0.86 \mathrm{bcd}$ & $0.85 \mathrm{cde}$ & $0.86 \mathrm{bcd}$ & $0.86 \mathrm{~B}$ \\
\hline Diagonal pits@90 & $0.84 \mathrm{ghi}$ & $0.90 \mathrm{de}$ & $0.85 \mathrm{ghi}$ & $0.86 f g h$ & $0.86 \mathrm{C}$ & $0.87 \mathrm{bcd}$ & $0.86 \mathrm{bcd}$ & $0.89 \mathrm{abc}$ & $0.85 \mathrm{de}$ & $0.87 \mathrm{~B}$ \\
\hline Double row strips & $0.82 \mathrm{i}$ & $0.85 \mathrm{f}-\mathrm{i}$ & $0.85 \mathrm{f}-\mathrm{i}$ & $0.85 \mathrm{ghi}$ & $0.84 \mathrm{C}$ & $0.81 \mathrm{f}$ & $0.81 \mathrm{f}$ & $0.88 \mathrm{bcd}$ & $0.81 \mathrm{ef}$ & $0.83 \mathrm{C}$ \\
\hline Trenches@120cm & $0.98 b c$ & $1.01 \mathrm{ab}$ & $1.03 \mathrm{a}$ & $0.98 b c$ & $1.00 \mathrm{~A}$ & $0.87 \mathrm{bcd}$ & $0.90 \mathrm{ab}$ & $0.93 \mathrm{a}$ & $0.87 \mathrm{bcd}$ & $0.89 \mathrm{~A}$ \\
\hline Mean $(\mathrm{K})$ & $0.88 \mathrm{C}$ & $0.92 \mathrm{~A}$ & $0.90 \mathrm{AB}$ & $0.90 \mathrm{~B}$ & & $0.85 \mathrm{~B}$ & $0.86 \mathrm{~B}$ & $0.89 \mathrm{~A}$ & $0.85 \mathrm{~B}$ & \\
\hline \multirow[t]{2}{*}{$\mathrm{LSD} \leq 0.05$} & \multicolumn{5}{|c|}{$\mathrm{K}=0.026 ; \mathrm{P}=0.015 \mathrm{~K} \times \mathrm{P}=0.035$} & \multicolumn{5}{|c|}{$\mathrm{K}=0.021 ; \mathrm{P}=0.013 ; \mathrm{K} \times \mathrm{P}=0.036$} \\
\hline & \multicolumn{10}{|c|}{ Number of millable canes $\left(\mathrm{m}^{2}\right)$} \\
\hline Pit planting@90 & $11.08 \mathrm{f}$ & $12.14 \mathrm{e}$ & $12.49 \mathrm{~cd}$ & $12.41 \mathrm{~d}$ & $12.03 \mathrm{~B}$ & $10.15 \mathrm{e}$ & $10.60 \mathrm{~b}$ & $10.37 \mathrm{~cd}$ & $10.48 \mathrm{bcd}$ & $10.40 \mathrm{~B}$ \\
\hline Diagonal pits@90 & $12.14 \mathrm{e}$ & $12.63 b c$ & $12.83 \mathrm{a}$ & $12.69 \mathrm{ab}$ & $12.57 \mathrm{~A}$ & $10.50 \mathrm{bcd}$ & $11.55 \mathrm{a}$ & $11.35 \mathrm{a}$ & $11.41 \mathrm{a}$ & $11.20 \mathrm{~A}$ \\
\hline Double row strips & $8.54 \mathrm{j}$ & $8.64 \mathrm{j}$ & $9.18 \mathrm{i}$ & $8.59 \mathrm{j}$ & 8.74D & $8.32 \mathrm{~g}$ & $9.12 \mathrm{f}$ & $9.29 \mathrm{f}$ & $9.18 \mathrm{f}$ & $8.98 \mathrm{D}$ \\
\hline Trenches@120cm & $9.15 \mathrm{i}$ & $10.13 \mathrm{~h}$ & $10.39 \mathrm{~g}$ & $10.29 \mathrm{~g}$ & $9.99 \mathrm{C}$ & $9.12 \mathrm{f}$ & $10.38 \mathrm{~cd}$ & $10.56 b c$ & 10.34de & $10.10 \mathrm{C}$ \\
\hline Mean $(\mathrm{K})$ & $10.23 \mathrm{D}$ & $10.88 \mathrm{C}$ & $11.22 \mathrm{~A}$ & $11.00 \mathrm{~B}$ & & $9.52 \mathrm{~B}$ & $10.41 \mathrm{~A}$ & $10.39 \mathrm{~A}$ & $10.35 \mathrm{~A}$ & \\
\hline $\mathrm{LSD} \leq 0.05$ & \multicolumn{5}{|c|}{$\mathrm{K}=0.094 ; \mathrm{P}=0.07 ; \mathrm{K} \times \mathrm{P}=0.16$} & \multicolumn{5}{|c|}{$\mathrm{K}=0.098 ; \mathrm{P}=0.12 ; \mathrm{K} \times \mathrm{P}=1.87$} \\
\hline
\end{tabular}


Table 4. Stripped cane yield (t/ha) and Agronomic efficiency of nutrients $(\mathrm{kg} / \mathrm{kg})$ as affected by various planting methods and $K$ application rates.

\begin{tabular}{|c|c|c|c|c|c|c|c|c|c|c|}
\hline \multirow[t]{3}{*}{ Planting methods } & \multicolumn{5}{|c|}{ 2014-15 (Plant crop) } & \multicolumn{5}{|c|}{ 2015-16(Ratoon crop) } \\
\hline & \multicolumn{10}{|c|}{ Potash application } \\
\hline & $\mathbf{0}$ & $100 \mathrm{~kg} \mathrm{ha}^{-1}$ & $200 \mathrm{~kg} \mathrm{ha}^{-1}$ & 300 kgha $^{-1}$ & $\operatorname{Mean}(\mathbf{P})$ & $\mathbf{0}$ & 100 kgha $^{-1}$ & $200 \mathrm{~kg} / \mathrm{ha}^{-1}$ & 300 kgha $^{-1}$ & $\operatorname{Mean}(\mathbf{P})$ \\
\hline & \multicolumn{10}{|c|}{ Stripped cane yield (t/ha) } \\
\hline Pit planting@90 & $93.69 f$ & $103.64 \mathrm{de}$ & $102.98 \mathrm{de}$ & $104.96 \mathrm{~cd}$ & 101.32B & $86.13 \mathrm{de}$ & $91.52 \mathrm{c}$ & $90.11 \mathrm{~cd}$ & $89.94 \mathrm{~cd}$ & $89.42 B$ \\
\hline Diagonal pits@90 & $103.72 \mathrm{de}$ & $113.65 \mathrm{a}$ & $108.12 \mathrm{a}$ & $109.32 b$ & $106.95 \mathrm{~A}$ & $93.36 \mathrm{bc}$ & $96.87 \mathrm{ab}$ & $98.22 \mathrm{a}$ & $97.24 \mathrm{ab}$ & $96.42 \mathrm{~A}$ \\
\hline Double row strips & $74.99 \mathrm{~h}$ & $78.38 \mathrm{~g}$ & $80.76 \mathrm{~g}$ & $78.29 \mathrm{~g}$ & $78.11 \mathrm{C}$ & $72.86 f$ & $73.80 \mathrm{f}$ & $74.87 f$ & $74.12 f$ & $73.91 \mathrm{C}$ \\
\hline Trenches@120cm & $94.64 f$ & 102.16de & $101.48 \mathrm{e}$ & $101.25 \mathrm{e}$ & $99.88 \mathrm{~B}$ & $84.53 \mathrm{e}$ & $92.76 \mathrm{c}$ & $91.52 \mathrm{c}$ & $90.37 \mathrm{c}$ & 89.79B \\
\hline Mean $(\mathrm{K})$ & $91.76 \mathrm{~B}$ & $98.21 \mathrm{~A}$ & $98.33 \mathrm{~A}$ & $97.95 \mathrm{~A}$ & & $84.22 \mathrm{~B}$ & $88.74 \mathrm{~A}$ & $88.68 \mathrm{~A}$ & $87.92 \mathrm{~A}$ & \\
\hline \multirow[t]{2}{*}{$\mathrm{LSD} \leq 0.05$} & \multicolumn{5}{|c|}{$\mathrm{K}=1.55 ; \mathrm{P}=1.91 ; \mathrm{K} \times \mathrm{P}=3.28$} & \multicolumn{5}{|c|}{$\mathrm{K}=1.07 ; \mathrm{P}=2.080 ; \mathrm{K} \times \mathrm{P}=3.77$} \\
\hline & \multicolumn{10}{|c|}{ Agronomic efficiency of nutrients } \\
\hline Pit planting@90 & $\begin{array}{c}--- \\
-1\end{array}$ & $99.45 a$ & $46.43 b$ & $37.54 \mathrm{bc}$ & $45.85 \mathrm{~A}$ & $\begin{array}{c}--- \\
-1\end{array}$ & $53.87 \mathrm{bc}$ & $19.88 \mathrm{de}$ & 12.70de & $21.61 \mathrm{AB}$ \\
\hline Diagonal pits@90 & ---- & $99.30 \mathrm{a}$ & $21.98 \mathrm{~cd}$ & $18.43 \mathrm{~cd}$ & $34.93 \mathrm{AB}$ & ---- & $59.70 \mathrm{ab}$ & $24.28 \mathrm{cde}$ & $12.92 \mathrm{de}$ & $24.23 \mathrm{AB}$ \\
\hline Double row strips & ---- & $33.90 \mathrm{bc}$ & $28.83 \mathrm{c}$ & $10.98 \mathrm{~cd}$ & $18.43 \mathrm{~B}$ & ---- & $13.17 \mathrm{de}$ & $10.07 \mathrm{de}$ & $4.21 \mathrm{de}$ & $6.86 \mathrm{~B}$ \\
\hline Trenches@120cm & ---- & $75.20 \mathrm{ab}$ & $34.18 b c$ & $22.02 \mathrm{~cd}$ & $32.85 \mathrm{AB}$ & ---- & $88.19 \mathrm{a}$ & $34.92 \mathrm{bcd}$ & $19.46 \mathrm{de}$ & $35.64 \mathrm{~A}$ \\
\hline Mean $(\mathrm{K})$ & ---- & $76.96 \mathrm{~A}$ & $32.86 \mathrm{~B}$ & 22.24B & & ---- & $53.73 \mathrm{~A}$ & $22.29 \mathrm{~B}$ & $12.32 \mathrm{BC}$ & \\
\hline $\mathrm{LSD} \leq 0.05$ & \multicolumn{5}{|c|}{$\mathrm{K}=12.43 ; \mathrm{P}=23.27 ; \mathrm{K} \times \mathrm{P}=35.70$} & \multicolumn{5}{|c|}{$\mathrm{K}=12.62 ; \mathrm{P}=26.33 ; \mathrm{K} \times \mathrm{P} 34.10$} \\
\hline
\end{tabular}

\begin{tabular}{|c|c|c|c|c|c|c|c|c|c|c|c|c|c|c|}
\hline \multirow[t]{2}{*}{$\begin{array}{l}\text { Trend } \\
\text { analysis }\end{array}$} & \multicolumn{2}{|c|}{ Leaf area index } & \multicolumn{2}{|c|}{$\begin{array}{c}\text { Leaf area } \\
\text { duration }\end{array}$} & \multicolumn{2}{|c|}{$\begin{array}{c}\text { Net assimilation } \\
\text { rate }\end{array}$} & \multicolumn{2}{|c|}{$\begin{array}{c}\text { Number of } \\
\text { millable canes }\end{array}$} & \multicolumn{2}{|c|}{$\begin{array}{c}\text { Stripped cane } \\
\text { yield (kg/ha) }\end{array}$} & \multicolumn{2}{|c|}{$\begin{array}{c}\text { Potassium Use } \\
\text { efficiency }\end{array}$} & \multicolumn{2}{|c|}{$\begin{array}{l}\text { Single cane } \\
\text { weight }(\mathrm{kg})\end{array}$} \\
\hline & 2014 & 2015 & 2014 & 2015 & 2014 & 2015 & 2014 & 2015 & 2014 & 2015 & 2014 & 2015 & 2014 & 2015 \\
\hline Linear & $*$ & $*$ & $*$ & $*$ & $*$ & $*$ & ns & ns & $*$ & ns & $*$ & $*$ & ns & ns \\
\hline Quadratic & $*$ & $*$ & $*$ & $*$ & * & * & * & * & $*$ & Ns & $*$ & * & $*$ & $*$ \\
\hline Cubic & ns & ns & ns & ns & $*$ & $*$ & $*$ & $*$ & Ns & Ns & Ns & Ns & $*$ & $*$ \\
\hline
\end{tabular}

$\mathrm{K}=$ Potash application; $\mathrm{P}=$ Planting methods; $*$ =Significant $(\mathrm{P}<0.05)$; ns =Non-significant; Capital alphabetical letters $(\mathrm{A}, \mathrm{B}, \mathrm{C}, \mathrm{D})$ with mean values represent the significant differences for main effects, while small alphabetical letters $(a, b, c \ldots)$ represent the interaction between planting methods and potash nutrition.

Interaction of 90 by $90 \mathrm{~d}$-pits and $200 \mathrm{~kg} / \mathrm{ha}$ favored the production of maximum stripped cane yield during both plant crop and ratoon crop year i.e. 113.64 and $98.22 \mathrm{t} / \mathrm{ha}$, respectively.

High potash use efficiency was recorded where $100 \mathrm{~kg} / \mathrm{ha}$ $\mathrm{K}_{2} \mathrm{O}$ was applied (Table 4). This trend realized additional cane yield of $76.96 \mathrm{~kg}$ for plant crop and $53.73 \mathrm{~kg}$ for ratoon crop with the application of $1 \mathrm{~kg}$ of potash nutrition in comparison with control and it was $14 \%$ more efficient than that of $200 \mathrm{~kg} / \mathrm{ha} \mathrm{K}_{2} \mathrm{O} .90$ by 90 pits showed high potassium use efficiency $(45.85 \mathrm{~kg} / \mathrm{kg})$ during the plant crop year; whereas it was achieved by using $120 \mathrm{ST}(35.64 \mathrm{~kg} / \mathrm{kg})$ during the ratoon crop year. Interaction of 90 by 90 pits with $100 \mathrm{~kg} / \mathrm{ha}(99.30 \mathrm{~kg} / \mathrm{kg})$ during the plant crop year and $120 \mathrm{ST}$ with $100 \mathrm{~kg} / \mathrm{ha}(88.19 \mathrm{~kg} / \mathrm{kg})$ during the ratoon crop year was effective in increasing KUE.

A linear increase in commercial cane sugar and total sugar recovery was found with the application of potash nutrition during both plant crop and ratoon crop year (Table 5). Planting methods and $\mathrm{K}$ did not make any significant contribution to the commercial cane sugar during the plant crop year; however, 90 by 90 pits increased $14.11 \%$ commercial cane sugar in ratoon crop year and $13.23 \%$ and $13.31 \%$, respectively, sugar recovery in both years. Sugar yield was substantially increased by the addition of potash nutrition up to $200 \mathrm{~kg} \mathrm{~K}_{2} \mathrm{O} / \mathrm{ha}$ in the plant crop year; however, it showed a linear trend in ratoon crop year. Diagonal pit plantation gave maximum production of sugar during both plant and ratoon years (15.08 and $12.65 \mathrm{t} / \mathrm{ha}$, respectively). Plantation of sugarcane in 90 by 90 d-pits and application of $100 \mathrm{~kg} \mathrm{~K} 2 \mathrm{O} /$ ha provided the maximum sugar yield during plant crop and ratoon crop year, i.e. 15.79 and $13.17 \mathrm{t} / \mathrm{ha}$, respectively (Table 5).

In addition to this, it was found that all of the treatments had higher BCR $(>1)$ in both plant crop and ratoon crop year, however the combined two years benefits revealed more economic benefits $(3678$ ) when sugarcane was planted in 120 ST with $100 \mathrm{~kg}$ /ha $\mathrm{K}$ nutrition which is followed by $3611 \$$ in 90 by $90 \mathrm{~d}$-pits with $100 \mathrm{~kg} / \mathrm{ha} \mathrm{K}_{2} \mathrm{O}$ (Table 6). 
Table 5. Commercial cane sugar, Total sugar recovery and total sugar yield as affected by various planting methods and $\mathrm{K}$ application rates.

\begin{tabular}{|c|c|c|c|c|c|c|c|c|c|c|}
\hline \multirow[t]{3}{*}{ Planting methods } & \multicolumn{5}{|c|}{ 2014-15 (Plant crop) } & \multicolumn{5}{|c|}{ 2015-16 (Ratoon crop) } \\
\hline & \multicolumn{10}{|c|}{ Potash application $\left(\mathrm{K}_{2} \mathrm{O}\right)$} \\
\hline & $\mathbf{0}$ & $100 \mathrm{~kg} \mathrm{ha}^{-1}$ & $200 \mathrm{~kg} \mathrm{ha}^{-1}$ & 300kgha ${ }^{-1}$ & Mean $(\mathbf{P})$ & $\mathbf{0}$ & 100 kgha $^{-1}$ & $200 \mathrm{~kg} / \mathrm{ha}^{-1}$ & 300 kgha $^{-1}$ & Mean $(\mathbf{P})$ \\
\hline & \multicolumn{10}{|c|}{ Commercial cane sugar } \\
\hline Pit planting@90 & 13.30 & 14.45 & 14.59 & 14.32 & $14.17 \mathrm{~A}$ & 13.49ef & $14.55 \mathrm{a}$ & $14.35 \mathrm{ab}$ & $14.05 \mathrm{a}-\mathrm{e}$ & $14.11 \mathrm{~A}$ \\
\hline Diagonal pits@90 & 13.58 & 13.89 & 14.31 & 13.72 & $13.87 \mathrm{~B}$ & $13.47 \mathrm{ef}$ & $14.15 a-d$ & $13.91 \mathrm{a}-\mathrm{e}$ & $14.03 \mathrm{a}-\mathrm{e}$ & $13.89 \mathrm{AB}$ \\
\hline Double row strips & 13.27 & 14.13 & 14.18 & 13.65 & $13.81 \mathrm{~B}$ & $13.82 b-f$ & $13.70 \mathrm{def}$ & $13.74 \mathrm{def}$ & $14.29 \mathrm{abc}$ & $13.89 \mathrm{AB}$ \\
\hline Trenches@120cm & 13.25 & 13.62 & 13.95 & 13.65 & $13.62 \mathrm{~B}$ & $13.74 b-f$ & $13.24 \mathrm{f}$ & $13.59 \mathrm{def}$ & $14.18 \mathrm{a}-\mathrm{d}$ & $13.69 \mathrm{~B}$ \\
\hline Mean & $13.35 \mathrm{C}$ & $14.02 \mathrm{~B}$ & $14.26 \mathrm{~A}$ & $13.83 \mathrm{~B}$ & & $13.63 \mathrm{~B}$ & $13.91 \mathrm{AB}$ & $13.90 \mathrm{AB}$ & $14.14 \mathrm{~A}$ & \\
\hline \multirow[t]{2}{*}{$\mathrm{LSD} \leq 0.05$} & \multicolumn{5}{|c|}{$\mathrm{K}=0.21 ; \mathrm{P}=0.10 ; \mathrm{K} \times \mathrm{P}=0.42$} & \multicolumn{5}{|c|}{$\mathrm{K}=0.252 ; \mathrm{P}=0.34 ; \mathrm{K} \times \mathrm{P}=0.64$} \\
\hline & \multicolumn{10}{|c|}{ Total sugar recovery } \\
\hline Pit planting@90 & $12.50 \mathrm{~g}$ & $13.59 \mathrm{ab}$ & $13.13 \mathrm{cde}$ & $13.72 \mathrm{a}$ & $13.23 \mathrm{~A}$ & $12.96 \mathrm{a}-\mathrm{d}$ & $13.56 \mathrm{a}$ & $13.51 \mathrm{ab}$ & $13.21 \mathrm{abc}$ & $13.31 \mathrm{~A}$ \\
\hline Diagonal pits@90 & $12.76 \mathrm{efg}$ & $13.13 \mathrm{cde}$ & $13.45 \mathrm{abc}$ & $12.90 \mathrm{def}$ & $13.06 \mathrm{~B}$ & $12.71 \mathrm{~cd}$ & $13.26 \mathrm{abc}$ & $12.80 \mathrm{~cd}$ & $13.37 \mathrm{abc}$ & $13.03 \mathrm{AB}$ \\
\hline Double row strips & $12.60 \mathrm{fg}$ & $13.28 \mathrm{bcd}$ & $13.33 \mathrm{abc}$ & $12.83 \mathrm{efg}$ & $13.01 \mathrm{~B}$ & $12.92 \mathrm{a}-\mathrm{d}$ & $12.93 \mathrm{a}-\mathrm{d}$ & $13.06 \mathrm{a}-\mathrm{d}$ & $13.35 \mathrm{abc}$ & $13.06 \mathrm{AB}$ \\
\hline Trenches@120cm & $12.46 \mathrm{~g}$ & $12.80 \mathrm{efg}$ & $13.11 \mathrm{cde}$ & $12.83 \mathrm{efg}$ & $12.80 \mathrm{C}$ & $12.42 \mathrm{~d}$ & $12.83 \mathrm{bcd}$ & $12.83 \mathrm{bcd}$ & $13.26 \mathrm{abc}$ & $12.84 \mathrm{~B}$ \\
\hline Mean & $12.58 \mathrm{~B}$ & $13.20 \mathrm{~A}$ & $13.25 \mathrm{~A}$ & $13.07 \mathrm{~A}$ & & $12.75 \mathrm{~B}$ & $13.14 \mathrm{AB}$ & $13.05 \mathrm{AB}$ & $13.30 \mathrm{~A}$ & \\
\hline \multirow[t]{2}{*}{$\mathrm{LSD} \leq 0.05$} & \multicolumn{5}{|c|}{$\mathrm{K}=0.20 ; \mathrm{P}=0.15 ; \mathrm{K} \times \mathrm{P}=0.38$} & \multicolumn{5}{|c|}{$\mathrm{K}=0.38 . ; \mathrm{P}=0.28 ; \mathrm{K} \times \mathrm{P}=0.71$} \\
\hline & \multicolumn{10}{|c|}{ Total sugar yield (t/ha) } \\
\hline Pit planting@90 & $12.46 \mathrm{~d}$ & $14.97 \mathrm{~b}$ & $15.02 \mathrm{~b}$ & $15.03 \mathrm{~b}$ & 14.37B & $11.15 \mathrm{de}$ & $12.41 \mathrm{bcd}$ & $12.17 \mathrm{~cd}$ & $11.88 \mathrm{cde}$ & 11.90B \\
\hline Diagonal pits@90 & $14.08 \mathrm{c}$ & $15.79 \mathrm{a}$ & $15.47 \mathrm{ab}$ & $14.99 \mathrm{~b}$ & $15.08 \mathrm{~A}$ & $11.87 \mathrm{cde}$ & $13.17 \mathrm{a}$ & $12.57 \mathrm{abc}$ & $13.00 \mathrm{ab}$ & $12.65 \mathrm{~A}$ \\
\hline Double row strips & $9.95 \mathrm{~g}$ & $11.08 \mathrm{ef}$ & $11.45 \mathrm{e}$ & $10.68 \mathrm{f}$ & $10.79 \mathrm{D}$ & $9.41 \mathrm{~h}$ & $9.59 \mathrm{~h}$ & $9.78 \mathrm{gh}$ & $9.90 \mathrm{gh}$ & $9.67 \mathrm{D}$ \\
\hline Trenches@120cm & $12.54 \mathrm{~d}$ & $13.91 \mathrm{c}$ & $14.15 \mathrm{c}$ & $13.82 \mathrm{c}$ & $13.60 \mathrm{C}$ & $10.50 \mathrm{fg}$ & $11.98 \mathrm{~cd}$ & $11.74 \mathrm{de}$ & $11.98 \mathrm{~cd}$ & $11.55 \mathrm{C}$ \\
\hline Mean & $12.26 \mathrm{C}$ & $13.94 \mathrm{~A}$ & $14.02 \mathrm{~A}$ & $13.63 \mathrm{~B}$ & & $10.73 \mathrm{~B}$ & $11.79 \mathrm{~A}$ & $11.56 \mathrm{~A}$ & $11.69 \mathrm{~A}$ & \\
\hline $\mathrm{LSD} \leq 0.05$ & \multicolumn{10}{|c|}{$\mathrm{K}=0.38 ; \mathrm{P}=0.34 ; \mathrm{K} \times \mathrm{P}=0.75$} \\
\hline
\end{tabular}

\begin{tabular}{|c|c|c|c|c|c|c|}
\hline \multirow[t]{2}{*}{ Trend analysis } & \multicolumn{2}{|c|}{ Commercial cane sugar } & \multicolumn{2}{|c|}{ Total sugar recovery } & \multicolumn{2}{|c|}{ Total sugar yield $\left(\right.$ tha $\left.^{-1}\right)$} \\
\hline & 2014 & 2015 & 2014 & 2015 & 2014 & 2015 \\
\hline Linear & $*$ & $*$ & $*$ & $*$ & $*$ & $*$ \\
\hline Quadratic & $*$ & $*$ & $*$ & Ns & $*$ & $*$ \\
\hline Cubic & ns & ns & ns & Ns & ns & ns \\
\hline
\end{tabular}

$\mathrm{K}=$ Potash application; $\mathrm{P}=$ Planting methods; *=Significant $(\mathrm{P}<0.05)$; ns =Non-significant; Capital alphabetical letters $(\mathrm{A}$, B, C, D) with mean values represent the significant differences for main effects, while small alphabetical letters $(\mathrm{a}, \mathrm{b}, \mathrm{c} .$.$) represent the interaction between planting methods and potash nutrition.$

Table 6. Net field benefits and benefit cost ratio by the application of potash under different planting methods in plant crop year (2013-14) and ratoon crop year 2015-16.

\begin{tabular}{|c|c|c|c|c|c|c|c|c|c|c|c|c|c|c|c|}
\hline \multirow[b]{2}{*}{ Treat. } & \multicolumn{7}{|c|}{ 2014-15 (Plant crop) } & \multicolumn{7}{|c|}{ 2015-16 (Ratoon crop) } & \multirow{2}{*}{$\begin{array}{c}\text { Two } \\
\text { year } \\
\text { profit } \\
\text { in US\$ }\end{array}$} \\
\hline & $\begin{array}{c}\text { Stripped } \\
\text { yield } \\
\text { (t/ha) }\end{array}$ & $\begin{array}{c}\text { Adjusted } \\
\text { yield } \\
\text { (t/ha) } \\
\end{array}$ & $\begin{array}{c}\text { Total } \\
\text { income } \\
\text { (Rs.) }\end{array}$ & $\begin{array}{c}\text { Total } \\
\text { cost } \\
\text { (Rs.) }\end{array}$ & $\begin{array}{c}\text { Benefit } \\
\text { cost } \\
\text { ratio } \\
\end{array}$ & $\begin{array}{c}\text { Net } \\
\text { benefits } \\
\text { (Rs.) }\end{array}$ & $\begin{array}{c}\text { Net } \\
\text { benefits } \\
\text { in } \$\end{array}$ & $\begin{array}{c}\text { Stripped } \\
\text { yield } \\
\text { (t/ha) }\end{array}$ & $\begin{array}{c}\text { Adjusted } \\
\text { yield } \\
\text { (t/ha) }\end{array}$ & $\begin{array}{c}\text { Total } \\
\text { income } \\
\text { (Rs.) }\end{array}$ & $\begin{array}{c}\text { Total } \\
\text { cost } \\
\text { (Rs.) } \\
\end{array}$ & $\begin{array}{c}\text { Benefit } \\
\text { cost } \\
\text { ratio }\end{array}$ & $\begin{array}{c}\text { Net } \\
\text { benefits } \\
\text { (Rs.) }\end{array}$ & $\begin{array}{c}\text { Net } \\
\text { benefits } \\
\text { in } \$ \\
\end{array}$ & \\
\hline $\mathrm{P}_{1} \mathrm{~K}_{0}$ & & & 389984 & 267014 & & 122970 & 1173 & & & 358516 & 150482 & & 208034 & & 3157 \\
\hline $\mathrm{P}_{1} \mathrm{~K}_{1}$ & 3.64 & & 431401 & 293694 & & 137707 & & & & 380952 & 175638 & & 205314 & & 3271 \\
\hline $\mathrm{P}_{\mathrm{I}} \mathrm{K}_{2}$ & 102.98 & 92.682 & 428654 & 316130 & 1.36 & 112523 & 1073 & 90.11 & 81.099 & 375082 & 197774 & 1.90 & 177308 & 1691 & 2764 \\
\hline $\mathrm{P}_{1} \mathrm{~K}_{3}$ & 104.96 & 464 & 436896 & 338922 & & 97973 & 934 & & & 374375 & 219706 & & 154669 & & 2409 \\
\hline $\mathrm{P}_{2} \mathrm{~K}_{0}$ & 103.72 & 93.348 & 431734 & 292921 & 1.47 & 138813 & 1324 & 93.36 & 84.024 & 388611 & 153374 & 2.53 & 235237 & 2243 & 3567 \\
\hline $\mathrm{P}_{2} \mathrm{~K}_{1}$ & 113.65 & 102.285 & 473068 & 319893 & & 153174 & 1461 & 96.87 & & 403221 & 177778 & 2.27 & 225443 & 2150 & 3611 \\
\hline $\mathrm{P}_{2} \mathrm{~K}_{2}$ & & 97.308 & 450049 & 340381 & & 109668 & 1046 & & & 408840 & 201018 & 2.03 & 207822 & 1982 & 3028 \\
\hline $\mathrm{P}_{2} \mathrm{~K}_{3}$ & 9.32 & & 455044 & 362861 & & 92183 & 879 & 97.24 & & 404761 & 222626 & 1.82 & 182135 & 1737 & 2616 \\
\hline $\mathrm{P}_{3} \mathrm{~K}_{0}$ & & & 312145 & 214968 & & & 927 & & & 303279 & 145174 & 2.09 & 158105 & 1508 & 2435 \\
\hline $\mathrm{P}_{3} \mathrm{~K}_{1}$ & 78.38 & 542 & 326256 & 239324 & 1.36 & 86932 & 829 & 73.8 & 66.420 & 307192 & 168550 & 1.82 & 138642 & 1322 & 2151 \\
\hline $\mathrm{P}_{3} \mathrm{~K}_{2}$ & & & 336163 & 262976 & & & 698 & & & 311646 & & & 119968 & 1144 & 1842 \\
\hline $\mathrm{P}_{3} \mathrm{~K}_{3}$ & & 461 & 325882 & 283988 & & 41893 & 399 & 12 & 08 & 308524 & 213378 & & 95146 & 907 & 1306 \\
\hline $\mathrm{P}_{4} \mathrm{~K}_{0}$ & 94.64 & 176 & 393939 & 223584 & 1.76 & 170354 & 1625 & 84.53 & 76.077 & 351856 & & 2.35 & 202014 & 1927 & 3552 \\
\hline $\mathrm{P}_{4} \mathrm{~K}_{1}$ & 102.16 & .944 & 425241 & 249592 & 1.70 & 175648 & 1675 & 92.76 & 83.484 & 386113 & 176134 & 2.19 & 209979 & 2003 & 3678 \\
\hline $\mathrm{P}_{4} \mathrm{~K}_{2}$ & 101.48 & 91.332 & 422410 & 272020 & 1.55 & 150390 & 1434 & 91.52 & 82.368 & 380952 & 198338 & 1.92 & 182614 & 1742 & 3176 \\
\hline $\mathrm{P}_{4} \mathrm{~K}_{3}$ & 101.25 & 91.125 & 421453 & 293928 & 1.43 & 127524 & 1216 & 90.37 & 81.333 & 376165 & 219878 & 1.71 & 156287 & 1490 & 2706 \\
\hline
\end{tabular}

$\mathrm{P}_{1} \mathrm{k}_{0}=90 \mathrm{~cm}$ spaced circular pits $+0 \mathrm{~kg} / \mathrm{ha} \mathrm{K}_{2} \mathrm{O} ; \mathrm{P}_{1} \mathrm{~K}_{1}=90 \mathrm{~cm}$ spaced circular pits $+100 \mathrm{~kg} / \mathrm{ha} \mathrm{K} \mathrm{K}_{2} ; \mathrm{P}_{1} \mathrm{~K}_{2}=90 \mathrm{~cm} \mathrm{spaced} \mathrm{circular} \mathrm{pits}+200 \mathrm{~kg} / \mathrm{ha} \mathrm{K} \mathrm{K}_{2} ; \mathrm{P}_{1} \mathrm{~K}_{3}=$ $90 \mathrm{~cm}$ spaced circular pits $+300 \mathrm{~kg} / \mathrm{ha} \mathrm{K}_{2} \mathrm{O} ; \mathrm{P}_{2} \mathrm{~K}_{0}=90 \mathrm{~cm}$ spaced circular pits in diagonal fashion $+0 \mathrm{~kg} / \mathrm{ha} \mathrm{K}_{2} \mathrm{O} ; \mathrm{P}_{2} \mathrm{~K}_{1}=90 \mathrm{~cm}$ spaced circular pits in diagonal fashion+100 kg/ha $\mathrm{K}_{2} \mathrm{O} ; \mathrm{P}_{2} \mathrm{~K}_{2}=90 \mathrm{~cm}$ spaced circular pits in a diagonal fashion $+200 \mathrm{~kg} / \mathrm{ha} \mathrm{K}_{2} \mathrm{O}$; $\mathrm{P}_{2} \mathrm{~K}_{3}=90 \mathrm{~cm}$ spaced circular pits in a diagonal arrangement $+300 \mathrm{~kg} / \mathrm{ha} \mathrm{K}_{2} \mathrm{O} ; \mathrm{P}_{3} \mathrm{~K}_{0}=90 \mathrm{~cm}$ spaced double row strips+0 kg/ha $\mathrm{K}_{2} \mathrm{O} ; \mathrm{P}_{3} \mathrm{~K}_{1}=90 \mathrm{~cm}$ spaced double row strips+100 kg/ha $\mathrm{K}_{2} \mathrm{O} ; \mathrm{P}_{3} \mathrm{~K}_{2}=90 \mathrm{~cm}$ spaced double row strips $+200 \mathrm{~kg} / \mathrm{ha} \mathrm{K}_{2} \mathrm{O} ; \mathrm{P}_{3} \mathrm{~K}_{3}=90 \mathrm{~cm}$ spaced double row strips $+300 \mathrm{~kg} / \mathrm{ha} \mathrm{K}_{2} \mathrm{O} ; \mathrm{P}_{4} \mathrm{~K}_{0}=120 \mathrm{~cm} \mathrm{spaced} \mathrm{trenches}+0 \mathrm{~kg} / \mathrm{ha} \mathrm{K}_{2} \mathrm{O} ; \mathrm{P}_{4} \mathrm{~K}_{1}=120$ cm spaced trenches $+100 \mathrm{~kg} / \mathrm{ha} \mathrm{K}_{2} \mathrm{O} ; \mathrm{P}_{4} \mathrm{~K}_{2}=120 \mathrm{~cm}$ spaced trenches $+200 \mathrm{~kg} / \mathrm{ha} \mathrm{K}_{2} \mathrm{O} ; \mathrm{P}_{4} \mathrm{~K}_{3}=120$ cm spaced trenches+300 kg/ha K $\mathrm{O}$; Two year profit in US\$: Combined profits of plant crop year (2013-14) and ratoon crop year (2015-16). 


\section{DISCUSSION}

Adequate fertilizers and proper planting methods are essential for all crops especially sugarcane because of its nutrient mining ability. Results revealed that sprouting capability of sugarcane was neither affected by potassium application nor by planting methods because sugarcane sett sprouting is dependent upon cane portion, availability of glucose to seedling, soil moisture and proper temperature (Sime, 2013). Potash nutrition has significant effect on tillering of sugarcane (Table 1). In general potassium is transported to the roots via diffusion; therefore applied potassium will be available in solution form in the rhizosphere. Previously it was reported that emergence of tillers is highly dependent on nutrient in the rhizosphere (Oliviria et al., 2004). Net assimilation rate of sugarcane was enhanced by potassium application because potassium speed up the nutrient flow and is directly involved in phloem loading and unloading (IPI, 2016) and fastens the speed of translocation of assimilates. Amongst the planting methods, 90 by 90 d-pits gave the maximum net assimilation rate; which may be due to extensive root system in pit plantation and better localized nutrient and water uptake by the cane plants in diagonal planting system.

Single cane weight was found be more in case of $100 \mathrm{~kg} \mathrm{~K}_{2} \mathrm{O}$ /ha and 120 ST during the plant crop year and $200 \mathrm{~kg} \mathrm{~K}_{2} \mathrm{O} / \mathrm{ha}$ during the ratoon crop year (Table 3 ). This means that during ratooning more potash nutrition is required for phloem loading and translocation of assimilates. The microenvironment available through trench planting may have favored the crop to get more girth, resultantly more cane weight was achieved. Number of millable canes $/ \mathrm{m}^{2}$ increased linearly by the potash application during both plant crop and ratoon crop year. These findings correlate with the findings of Hussain et al. (2017), who stated that increased application of potash has a positive and linear effect on number of millable canes. Moreover, 90 by $90 \mathrm{~d}$-pits produced more number of millable canes, which may be due to more seed rate per square meter than that of other plantation schemes. Higher stripped cane yield was also produced by 90 by 90 d-pits that may be related to higher seed rate in this planting method than that of 120 ST; where canes with more weight were obtained. Furthermore, high stripped cane yield in 90 by 90 d-pits may be due to efficient use of resources and environmental factors to explore the genetic potential of cane during the developmental period (Tena et al., 2016). Potash nutrition enhanced the stripped cane yield of sugarcane during both the years of study. Under field condition, stripped cane yield response to potash nutrition depends upon the soil reserves of potassium whether these are exchangeable or nonexchangeable resources (Kingston et al., 2009). Moreover, stripped cane yield in ratoon crop in sub-continent is believed to be in between 50-55 t/ha as compared to planted crop yield which is above $70 \mathrm{t} / \mathrm{ha}$ (Lal and Singh, 2008). The ratoon crop yield can well be managed by appropriate planting methods like pit plantation and trench plantation in combination with the Potash nutrition as depicted by the results because bud initiation and sprouting can be bettered by proper uptake of nutrients (Shukla et al., 2009b). The obtained higher potassium use efficiency was increased with $100 \mathrm{~kg} \mathrm{~K}_{2} \mathrm{O} / \mathrm{ha}$ during both the years, as each additional $76.96 \mathrm{~kg}$ plant biomass during plant crop year and $53.54 \mathrm{~kg}$ during ratooning, were produced with the input of $1 \mathrm{~kg}$ of potash application. In case of planting methods, 90 by 90 pits gave maximum potassium use efficiency during the plant crop year and trenches gave maximum potassium use efficiency during the ratoon crop year. Localized application in pits and trenches favored the efficient utility of nutrients especially macronutrients $(\mathrm{K})$, as they are placed in the closest vicinity of the roots (Yadav, 2004).

Sugar recovery of sugarcane was improved by all level of potash application during the plant crop and ratoon crop year, because of the fact that sugarcane juice is abundant in ash contents and ash contents are abundant in potassium. Therefore, an application of potash fertilizer to the already soil deficient in potassium, will increase the polarity $\%$ and reduce the fiber\% in the juice (a significant reduction in starch contents) (Mayer and Wood, 2001). Moreover, it has been stated that potassium is as an essential element in the productivity and sugar recovery of sugarcane ratoon (Weber et al., 2002). Sugar yield is the product of commercial cane sugar and stripped cane yield; therefore, a treatment combination of $100 \mathrm{~kg} \mathrm{~K} 2 \mathrm{O} / \mathrm{ha}$ and 90 by 90 d-pits gave the maximum yield during the plant crop as well as ratoon crop year (Table 5).The higher number of millable canes and cane weight resulted in higher stripped cane yield and hence, contributed to higher economic benefits. Combined net benefits of both plant and ratoon year revealed that 120 ST $+100 \mathrm{~kg} / \mathrm{ha} \mathrm{K}_{2} \mathrm{O}$ gave maximum field benefits/net returns of $3678 \$$. The reason may be that land preparation, mechanical pit plantation, the shaping of pits and sowing of 30 double budded setts during the plant crop year was expensive and did not contribute significantly to cover the costs with appreciable benefits. Hence even stripped cane yield is higher in case of 90 by 90 d-pits than all other systems, however, 120 ST was found more economical due to less cost of field preparation for trench planting.

Conclusion: It can be concluded that K application at 100 $\mathrm{kg} / \mathrm{ha}$ is sufficient for attaining higher sugar recovery. Amongst different planting methods, $120 \mathrm{~cm}$ spaced trench planting has been found to be economically feasible for plant and ratoon planting of sugarcane crop.

Acknowledgment: Authors are grateful for the financial support by Higher Education Commission of Pakistan for this research. 


\section{REFERENCES}

Abbas, H.T., S.T. Sahi, A. Habib and S. Ahmed. 2016. Laboratory evaluation of fungicides and plant extracts against different strains of Colletotrichum falcatum the cause of red rot of sugarcane. Pak. J. Agri. Sci. 53:181186.

Ali, H., I. Ahmad, S. Hussain, M. Irfan, A. Areeb and R.N. Shabir. 2018. Basal application of potassium nutrition enhances cane yield, juice quality and net returns of sugarcane (Saccharum officinarum L.). Pak. J. Agri. Sci. 55:321-329.

CIMMYT. 1988. From agronomic data to farmers recommendations: An economics training manual. CIMMYT, Mexico. D.F.

Chattha, M.U., A. Ali and M. Bilal. 2007. Influence of planting techniques on growth and yield of spring planted sugarcane (Saccharum officinarum L.). Pak. J. Agri. Sci. 44:452-456.

Chohan, M., U.A. Talpur, R.N. Pahnwar and S. Talpur. 2013. Effect of inorganic NPK different levels on yield and quality of sugarcane plant and ratoon crop. Int. J. Agron. Prod. 4:3668-3674.

El-Tilib, M.A., M.H. Elnasikh and E.A. Elamin. 2004. Phosphorus and potassium fertilization effects on growth attributes and yield of two sugarcane varieties grown on three soil series. J. Plant Nutr. 27:663-699.

Ehsanullah, K. Jabran, M. Jamil and A. Ghafar. 2011. Optimizing the sugarcane row spacing and seeding density to improve its yield and quality. Crop Environ. 2:1-5.

Ghaffar, A., Ehsanullah, N. Akbar, S.H. Khan, R. Qamar, K. Jabran, A. Iqbal and M.A. Ali. 2012. Effect of trench spacing and micronutrients on growth and yield of sugarcane (Saccharum officinarum L.). Aust. J. Crop. Sci. 6:1-9.

Govt. of Pakistan. 2016. Economic survey of Pakistan 201516. The government of Pakistan, Economic Affairs Division, Ministry of Finance, Islamabad, Pakistan.

Hunsigi, G. 2011. Potassium management strategies to realize high yield and quality of sugarcane. Karnataka J. Agric. Sci. 24:45-47.

Hunt, R. 1978. Plant growth analysis. The institute of biological sciences. Edward Arnold (Pub.) Ltd. 96:8-38.

Hussain, S., M. Anwar, S. Hussain, Z. Akram, M. Afzal and I. Shabbir. 2017. Best suited timing schedule of inorganic NPK fertilizers and its effect on qualitative and quantitative attributes of spring sown sugarcane (Saccharum officinarum L.). J. Sau. Soc. Agric. Sci. 16:66-71.

IPI (International potash Institute). 2016. Potassium in plant production. Available online with updates at https://www.ipipotash.org/en/publications/detail.php?i= 73.

Kingston, G., M.C. Anink, B.M. Clift and R.N. Beattie. 2009. Potassium management for sugarcane on base saturated soils in northern New South Wales. Proc. Aust. Soc. Sugar Cane Technol. 31:186-194.

Krauss, A. 2001. Potassium and biotic stress. Proceedings of the $1^{\text {st }}$ FAUBA-FERTILIZAR-IPI workshop on potassium in Argentina's agricultural systems, 20-22 November 2001, Buenos Aires; pp.281-293.

Lal, M and A.K. Singh. 2008. Multiple ratooning for high cane productivity and sugar recovery.

Proceedings of National Seminar on Varietal Planning for Improving Productivity and Sugar Recovery in Sugarcane; pp.62-68.

Meyer, J.H. and R.A. Wood. 2001. The effects of soil fertility and nutrition on sugarcane quality: A review. Proc. S. Afr. Sug. Technol. Assoc. 75:242-247.

Nalewade, S.M., K. Mehta and A.K. Sharma. 2018. Planting techniques: A review. Contemp. Res. Ind. 3:98-104.

Oliveira, R.H., C.A. Rosolem and R.M. Trigueiro. 2004. Importance of mass flow and diffusion on the potassium supply to cotton plants as affected by soil water and potassium. Rev. Bras. Cienc. Solo. 28:439-445.

Sime, M. 2013. The effect of different cane portions on sprouting, growth and yield of sugarcane (Saccharum spp. L.). Int. J. Sci. Res. 3:1-3.

Singh, G.B. 2002. Management of plant population in sugarcane for higher productivity and quality. Proceedings of international symposium on food, nutrients and economic security through diversification in sugarcane production and processing systems; pp.918.

Singh, K.P., A. Suman, P.N. Singh and M. Lal. 2007. Yield and soil nutrient balance as on sugarcane plant-ratoon system with conventional and organic nutrient management in sub-tropical India. Nutr. Cycl. Agroecosys. 79:209-219.

Singh, K., M.S. Bhullar, K. Singh and S.K. Uppal. 2009. Effect of planting techniques for spring planted Sugarcane. J. Res. Punjab Agric. Uni. 46:137-139.

Shukla, S.K., R.L. Yadav, P.N. Singh and I. Singh. 2009. Potassium nutrition for improving stubble bud sprouting, dry matter partitioning, nutrient uptake and winter initiated sugarcane (Saccharum spp. hybrid complex) ratoon yield. Eur. J. Agron. 30:27-33.

SSSP. 1994. Efficient use of plant nutrients. Proceedings of Fourth National Congress of Soil Science, Islamabad, May 24-26, 1992. Soil Sci. Soc. Pak; p.3.

Steel, R.G.D., J.H. Torrie and D.A. Dicky. 1997. Principles and Procedures of Statistics: A biometrical approach, $3^{\text {rd }}$ Ed. McGraw Hill Book Int. Co., Singapore; pp.204-227.

Tena, E., F. Mekbib, H. Shimelis and L. Mwadzingeni. 2016. Sugarcane production under smallholder farming 
systems: Farmers preferred traits, constraints and genetic resources. Cogent Food Agric. 2:1-15.

Weber, H., E. Daros, J.L.C. Zambon, O.T. Ido and J.D. Barela. 2002. Ratoon sugarcane productivity recovering with NPK fertilization. Sci. Agrar. 2:73-77.
Yadav, R.L. 2004. Enhancing efficiency of fertilizer $\mathrm{N}$ use in sugarcane by ring-pit method of planting. Sugar Tech. 6:169-171. 